\title{
Reusable Modeling of Pulsed Doppler Radar Seeker for Coherent Video Signal Simulation
}

\author{
Jing Zhao ${ }^{1, a^{*}}$, Shenshen Wang ${ }^{1, b}$, Wanfang Che ${ }^{1}$ and Fangnian Wang ${ }^{2, c}$ \\ ${ }^{1}$ Key Lab of Complex Aviation System Simulation, Beijing, 100076, China \\ ${ }^{2}$ Air Force Airborne Academy, Guilin, 541003, China \\ ajznudt@126.com, bwangshenshen0616@163.com, 'wfangnian@126.com
}

Keywords: Reusable Model; Radar Seeker; Coherent Video Signal; Design Pattern

\begin{abstract}
A reusable framework of pulsed Doppler radar seeker (PDRS) is established for coherent video signal simulation, which provides an efficient tool to its design and evaluation. General PDRS configurations are described with emphasis on performance limitations. The discussions on the key parts of PDRS are self-contained and provide sufficient detail to allow a clear understanding of principles involved, which are depicted in a series of UML class diagram. Augmented proportional navigation law is used to generate the acceleration demands, while the bank-to-turn control command to obtain the fin-angle demand and the six degree of freedom (6-DOF) missile motion solution can then be accomplished by using missile aerodynamic characteristics. An air defense warfare scenario is designed to validate coherent video modeling. The 6-DOF trajectory simulation is also considered. The electronic countermeasures are also taken into account. Results show that this PDRS coherent video model is accurate for 6-DOF trajectory simulation, and the closed loop simulation offers an effective and practical way to design parameters and evaluate performance of missile.
\end{abstract}

\section{Introduction}

Pulsed Doppler radar seeker (PRDS) is widely deployed in modern surface-to-air missile (SAM) to play as a crucial line-of-sight (LOS) angle sensitive device [1]. PDRS utilizes a coherent pulsed transmitter that uses the Doppler effect, which is associated with the radical component of relative radar-target velocity, to select targets having particular radical velocities in clutter and receiver noise. Guidance law generator is fed by those PDRS outputs mainly including LOS angles and angular rates with regard to the designated target. To help design its parameters and evaluate performance, several testing approaches containing mathematical simulation, hardware-in-the-loop simulation and field range tests are needed. The mathematical simulation provides an efficient and low-cost way of design and then get it's widely usage for those PDRS designers. However, a need rises of establishing adequately accurate mathematical models of each part. The parts involved are antenna, transmitter, receiver, signal processor, data processor and main controller [2]. Modeling accuracy can directly affect the precision, thus one has to model them with emphasis on performance limitations [3].

Conventional functional-level model of PDRS, which is widely used due to its simpleness, can't reflect the process of electromagnetic wave propagation and scattering, as well as the coherent processing in receiver and consecutive digital signal processor, thus it is inefficient in accurate PDRS simulation, especially in those cases of ECM [4]. On the other hand, coherent video signal simulation can describe the process of wave transmission, propagation, back scattering, antenna receiving, receiver processing, digital signal processing and maybe following data processing $[5,6]$, in a comprehensive way. This paper is dedicated to the design of reusable PDRS model for coherent video signal simulation. A series of UML class diagram are drawn to clarify the design principles, while case studies are finished to validate the modeling accuracy and reusability. 


\section{PDRS Configurations}

A basic decision that must be made in PRDS design is the selection of the pulse repetition frequency (PRF). PRDS can be categorized as low, medium and high PRF, according to whether Doppler (low PRF), range (high PRF), or both of them are ambiguous (medium PRF).

The choice among the various waveforms in PDRS mainly depends on its application. LOS angles and rates are necessary to obtain acceleration demands, while slant range and range rate are somewhat unimportant, though they may be needed in the target tracking loop and other advanced guidance law. A high-PRF waveform provides a clutter-free target detection region that generally facilitates detection of closing targets, which is of great importance in SAM. HPRF designs require complicated methods for resolving ambiguities of range, however, this is not necessary for SAM's tracking loop, as only one Doppler filter may be needed for tracking once the target is acquired, which means that we can select targets that have particular radial velocities in heavy ground or sea clutter at the absence of range/range rate measurements. PDRS configurations utilizing a HPRF waveform are accomplished, as depicted in Figure 1.

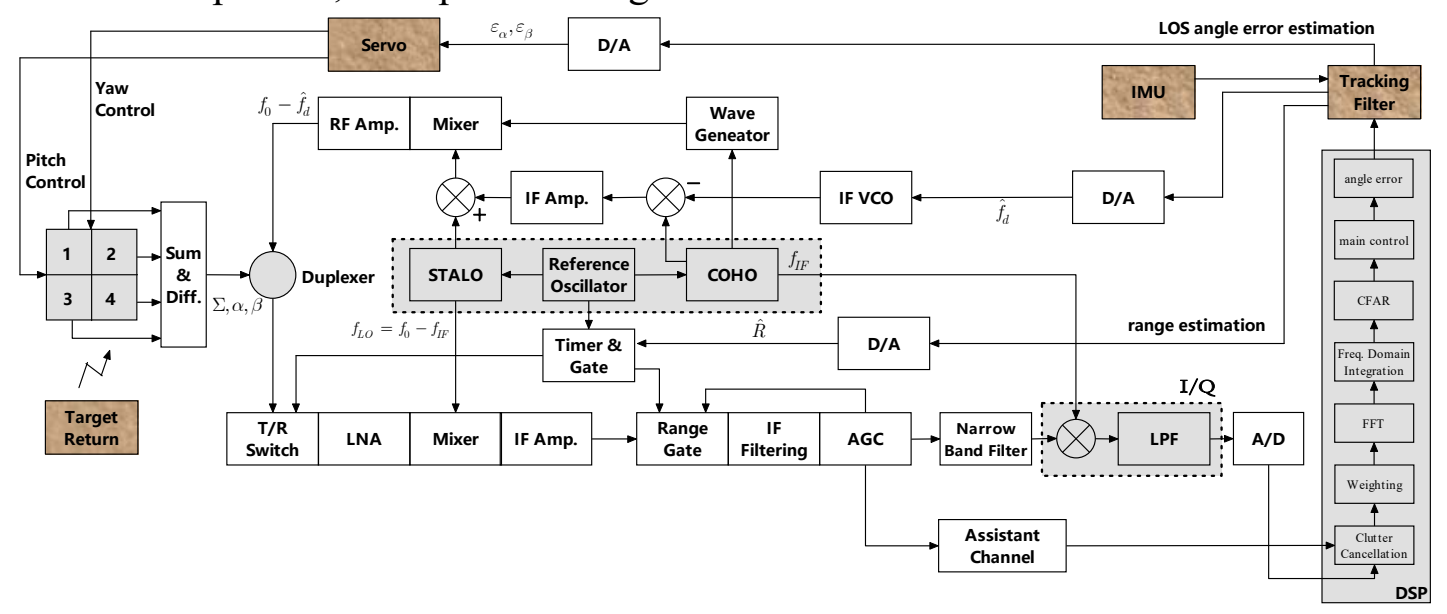

Fig. 1. PDRS configurations.

\section{Reusable modelling of PDRS}

PDRS transmits the coded radio frequency electromagnetic wave, propagated in atmosphere and reflected by existing objects, while receives backscatter returns via an antenna duplexer. Those returns are preceded in a digital signal processor to help extract missile-to-target motion including relative range, range rate, LOS angles and rates. Gimbal angle errors are also obtained by monopulse processing, and then sent to servo system to sustain a closed-loop angular tracking.

From the object-oriented programming (OOP) viewpoint, we treat PDRS as a combination of several key parts including antenna, transmitter, receiver, signal processor, recognition, data processor and main controller, as depicted in Figure 2. Some key parts are discussed as followed. At the same time, every part in PDRS has its own configuration. Several key application programming interfaces (API) are designed and reserved, as initialization, transmission, receiving, etc. 


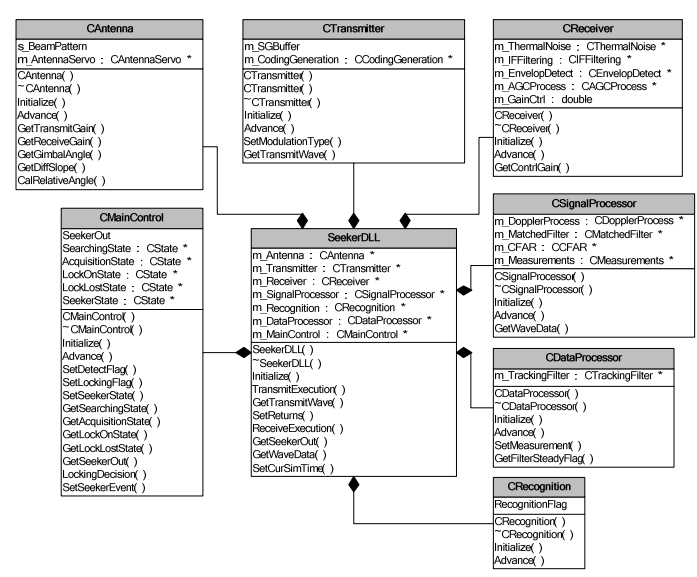

Fig. 2. PDRS static structure.

Antenna model is fundamental to PDRS simulation. Electromagnetic wave is transmitted and received by seeker antenna, and servo system is used to stabilize antenna boresight pointing to target despite of rigid missile body motion. After making an abstract of antenna system, we can describe it in Figure 3.

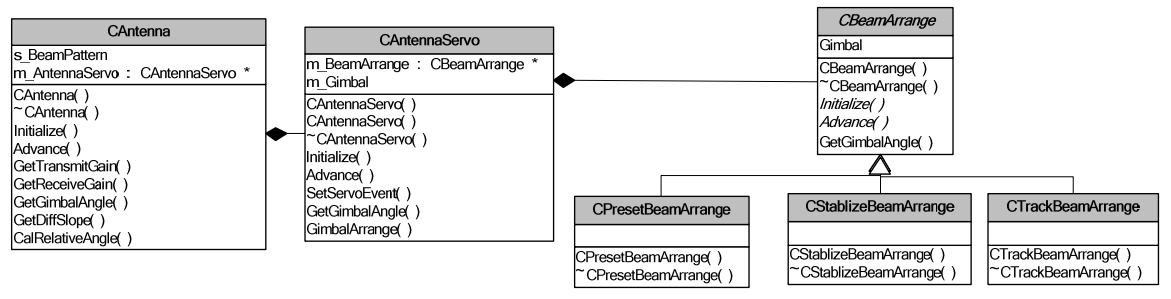

Fig. 3. Antenna model design.

By using Strategy Pattern, antenna servo system is designed as a virtual class, in which we can execute the presetting, stabilization and tracking, respectively.

A simplified antenna pattern of single beam is established by using Sinc function. Once antenna pattern of a single beam is finished, one can get gains of both sum and difference channels by proper combination and the gains can be used to modulate target returns and abstract gimbal angle errors with regard to LOS.

PDRS utilizes a coherent transmitter to transmit coded radio frequency wave in directivity. The coherent pulse train signals returned from targets illuminated by PDRS are processed in a set of narrowband filters that coherently integrate the signals to which they are tuned. Transmitter can be categorized as single pulse, linear frequency modulation and pulse code modulation, according to the intra-pulse modulation type. The Strategy Pattern is utilized here again, as in Figure 4.

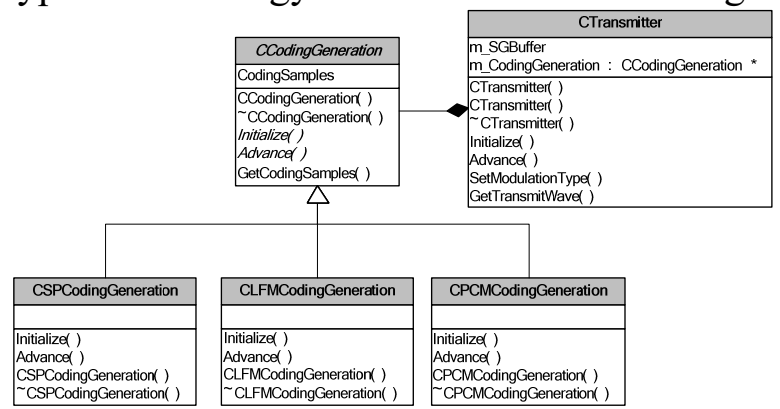

Fig. 4. Transmitter model design.

As shown in Figure 1, PDRS receiver converts target returns to intermediate frequency (IF) by the down-conversion processing. Range gate is utilized to select target return while avoid outside clutter and noise. IF filter amplifies echoes to a certain level for the consecutive narrow band filtering and I/Q demodulation. Different from conventional active radar seeker, HPRF design utilizes the narrow band filtering to extract and track only the center spectrum line, in comparison to the matched filter. A quasi-continuous waveform is obtained after narrow band filtering, and the following DSP carries out detection and tracking both in frequency domain. 


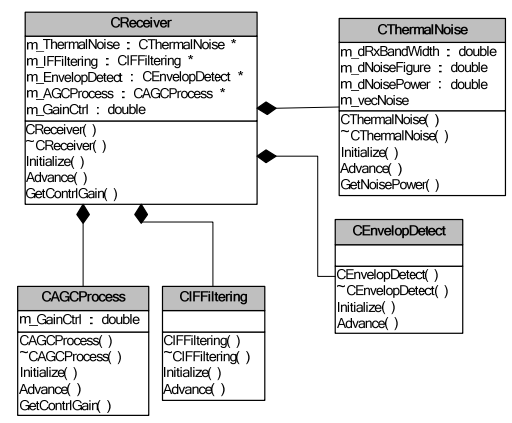

Fig. 5. Receiver model design.

Functions of digital signal processor include clutter cancellation, time domain weighting, FFT, frequency domain coherent/incoherent integration, CFAR, angle error measuring, etc. Signal processor plays as a crucial role in PDRS to detect and track target in clutter, noise and may be ECM, and its model design is shown in Figure 6. The HPRF design suffers from high range ambiguity and in PDRS we usually don't need a slant range track loop. So missile-to-target range won't be measured. Closing rate can be measured by tracking the centre spectrum line in the bank of contiguous Doppler filters, which is usually referred to as the high-low Doppler filter. While the angle errors of line-of-sight with regard to antenna boresight, i.e. gimbal angle errors, can be measured by monopulse processing.

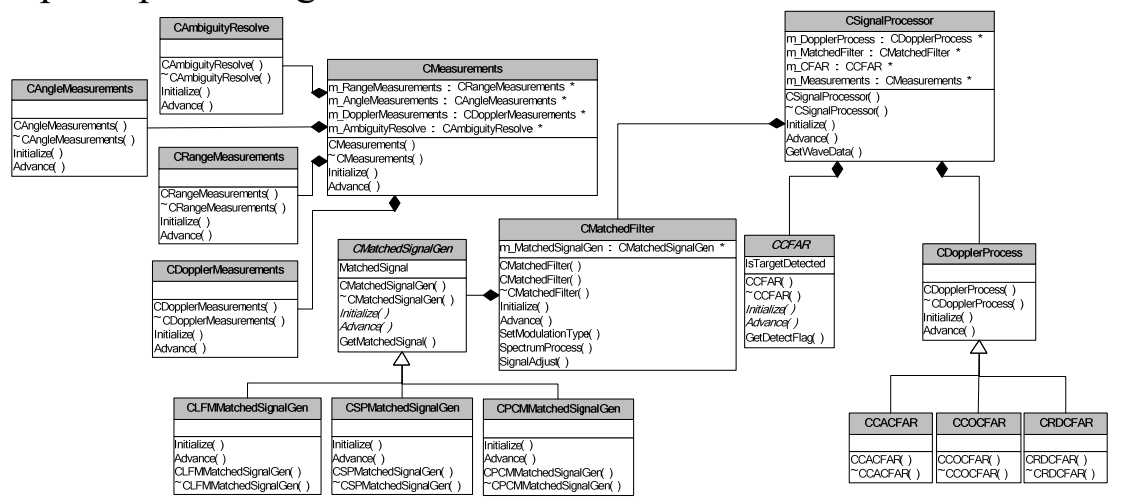

Fig. 6. Signal Processor model design.

Main controller. Main controller plays as a brain role in PDRS to change seeker state properly in clutter, noise and ECM background. Four state, searching, acquisition, tracking and memory tracking, are transferred to each other under certain conditions, as seen in Figure 7. Note that we can use State Pattern to describe its proceeding.

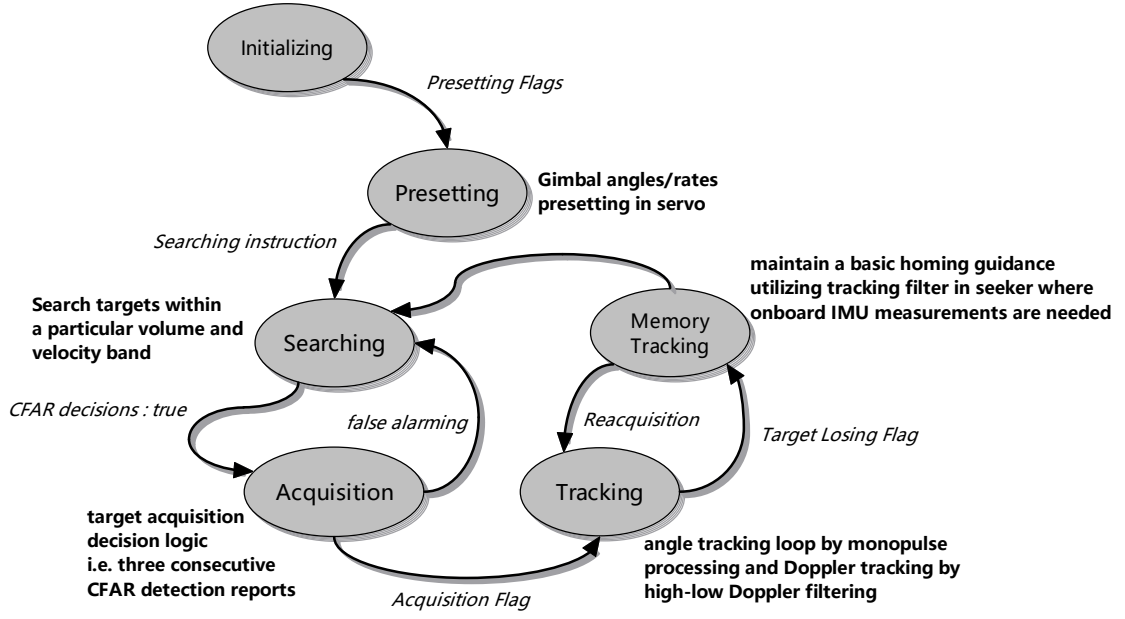

Fig. 7. PDRS status switching logic. 


\section{Case study}

A typical air defence scenario is designed. As we focus on the terminal phase of engagement, the midcourse guidance utilizing inertial navigation solution is not considered. Besides, the six degree of freedom (6-DOF) trajectory simulation is carried out to help evaluate performance of PDRS comprehensively.

The terminal phase of surface-to-air engagement puts forward a high requirement on missile guidance and control loop, depicted in Figure 8, as one has to take some key factors into account including high-speed, low-altitude, stealth, maneuver, onboard ECMs, autopilot tag, fin-angle demand, aerodynamic characteristics, etc. The seeker outputs, i.e. missile-to-target relative motion measurements, are converted into three axes acceleration demands by using specified guidance law, such as the augmented proportional navigation law (APN) used in this paper. Considering real-time missile flying status, mainly its body attitude, a control command unit generate the control instruction transferred to the following execution device, i.e. a tail fin to stabilize body while guide the missile to the designated target according to the input acceleration demand. Tail fin responses the steering angle commands to achieve the actual fin angles. Once fin angles are changed, the interceptor SAM is aerodynamically controlled, and a 6-DOF closed loop missile trajectory simulation is finally accomplished. A bank-to-turn (BTT) control law is usually adopted as it can guide missile to the designated target while stabilize the body. Real-time missile flying status that includes the acceleration in both pitch and yaw axis and body rotation rates are needed. This control is somewhat complicated and can be found in [7,8]. Tail fins on missile body response to the above BTT control command to achieve actual fin angle in yaw, pitch and roll channel, respectively. Aerodynamic characteristic of SAM changes accordingly and so the missile flying status.

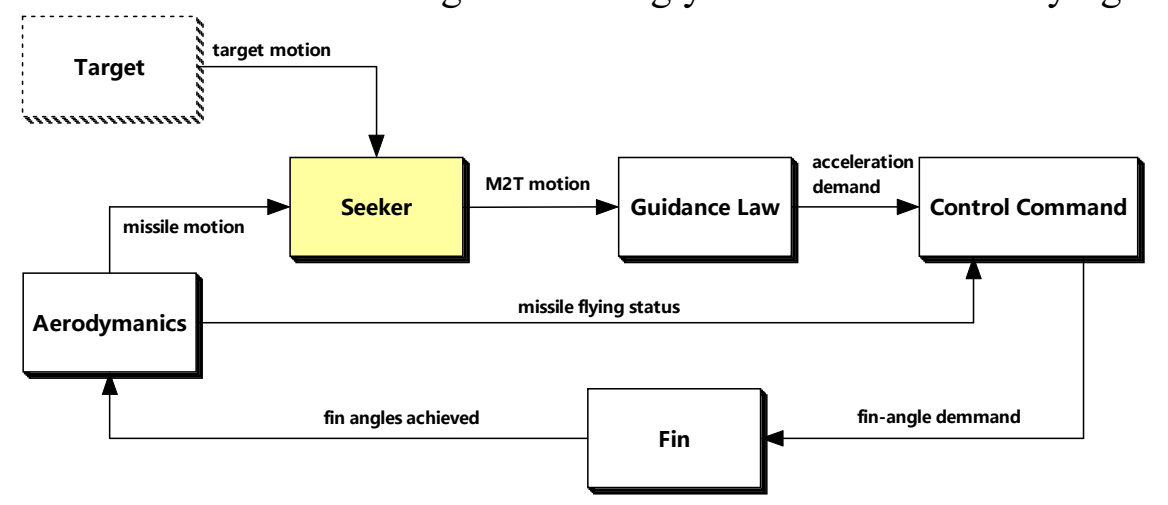

Fig. 8. Guidance and control loop.

Relative motion in local-level reference frame (LLF) is shown in Figure 9, where missile attitude (i.e. pitch, yaw, roll angle) is also described. Missile-to-target motion is clearly formulated in LLF.
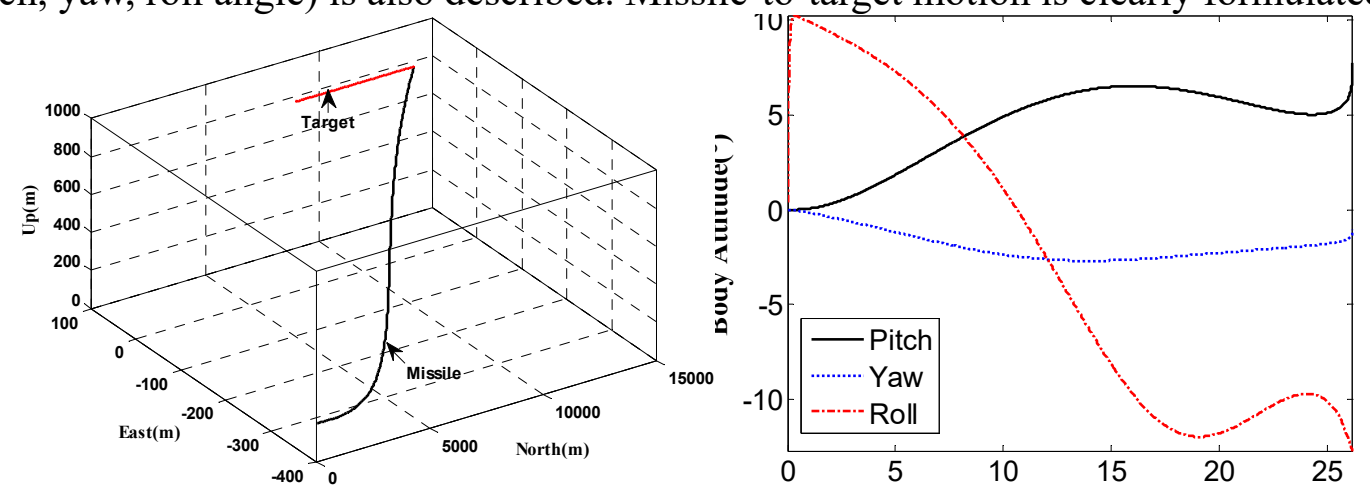

Fig. 9. Missile-to-target relative motion.

Coherent video modelling results of radar seeker are shown. The normalized spectrum of I/Q coherent video samples, as seen in left of Figure 10, reflects clearly the Doppler shift due to the radical speed of missile-to-target. Using Doppler filtering in DSP, one can obtain this Doppler shift used in target velocity tracking loop. The SNR curve is also shown on the right. Note that in a 
seeker utilizing HPRF, eclipsing loss occurs intermittently and measurements are not available periodically, which increases the difficulty in continuous target tracking.
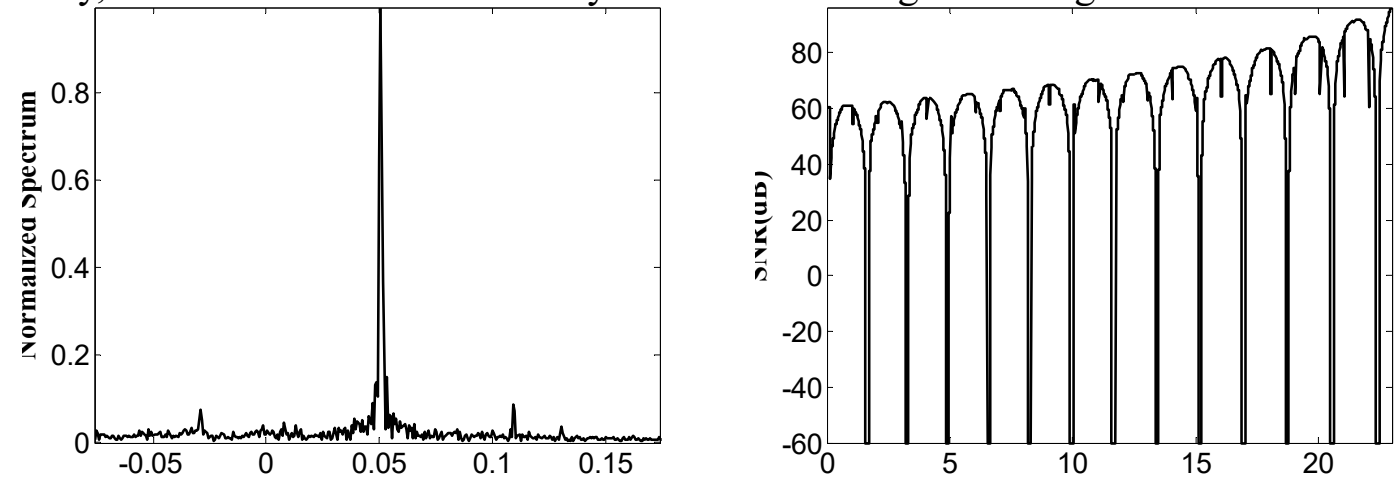

Fig. 10. Spectrum (left) and SNR (right).

PDRS gimbal angle error measurements are seen in Figure 11. By using monopulse processing techniques, one can extract those angle errors of target with regard to antenna boresight, i.e. gimbal. High measuring accuracy (approx. 1mrad) is acquired, which provides an advantage of homing guidance precision and may be a better rejection of chaff and towed radar active decoys (TRAD).
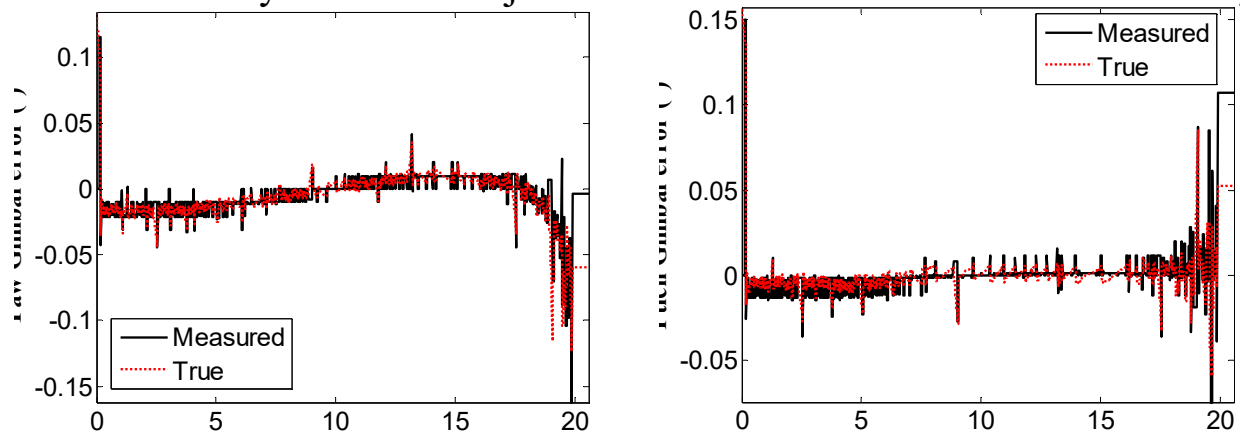

Fig. 11. Gimbal angle error (left: yaw, right: pitch).

In ECM case e.g. velocity gate pull off (VGPO) jamming, the seeker Doppler tracking loop is interrupted, and the closing rate of missile-to-target couldn't be correctly extracted, and the homing guidance precision is thus affected (the miss distance increases from $2.48 \mathrm{~m}$ to $56.95 \mathrm{~m}$ ). Note that the PDRS coherent video model provides an advantage of demonstrating the great impact made by the interferences on seeker homing accuracy and guidance precision [10].

\section{References}

[1] Y. M. Sun, J. Chen, C. H. Guo et al., "The advanced simulation system for MMW imaging radar seeker on-board air-to-air missile", 2006 the 8th International Conference on Signal Processing, pp. 2854-2857.

[2] H. F. Li, and M. Zhao, "The System Research of PD Radar's Seeker Based on Multiple Target", 2010 International Conference on Information, Electronic and Computer Science, pp. 2010-2015.

[3] Z. S. Jia, and X. C. Si, "Analysis on the Tracking Performance of Active Radar Seeker under the Condition of Coherent Interference", 2009 IEEE International Conference on Intelligent Computing and Intelligent Systems, pp. 418-422.

[4] A. Farooq, and D. J. N. Limebeer, "Optimal trajectory regulation for radar imaging guidance," Journal of Guidance Control and Dynamics, vol. 31, no. 4, pp. 1076-1092, Jul-Aug, 2008.

[5] J. B. Zou, K. Gao, and E. Y. Zhang, "Research on Angle Measurement Model of Anti-radiation Missiles PRS under Coherent Decoys," Przeglad Elektrotechniczny, vol. 88, no. 3B, pp. 18-21, 2012. 
[6] P. Vora, P. K. Tiwari, R. N. Bhattacharjee et al., "Radio frequency seeker modelling and seeker filter design,” Defense Science Journal, vol. 55, no. 3, pp. 337-348, Jul, 2005.

[7] C. Rago, R. K. Mehra. "Robust adaptive target state estimation for missile guidance using the interacting multiple model Kalman filter". IEEE Position Location and Navigation Symposium. 13 16 March, 2000. 355 362.

[8] Y. T. Zhao, Y. A. Hu, and J. X. Lin, "Research on the Handover of the Compound Guidance for the Anti-ship Missile beyond Visual Range", International Conference on Computing, Information and Control (ICCIC 2011), pp. 224-231.

[9] J. Muller, and J. Plorin, "LFK hardware-in-the-loop facility for missile development and evaluation," Technologies for Synthetic Environments: Hardware-in-the-Loop Testing Vi, Proceedings of the Society of Photo-Optical Instrumentation Engineers (Spie), pp. 65-72, 2001.

[10] C. Inaebnit, M. A. John, and U. Aulenbacher, "Radar-target signatures from mmw-measurement platform," Passive Millimeter-Wave Imaging Technology Vi and Radar Sensor Technology Vii, Proceedings of the Society of Photo-Optical Instrumentation Engineers, pp. 235-243, 2003. 